\title{
Increased risk of pseudoprogression among pediatric low-grade glioma patients treated with proton versus photon radiotherapy
}

\author{
Ethan B. Ludmir, ${ }^{\oplus}$ Anita Mahajan, Arnold C. Paulino, Jeremy Y. Jones, Leena M. Ketonen, \\ Jack M. Su, David R. Grosshans, Mary Frances McAleer, Susan L. McGovern, \\ Yasmin A. Lassen-Ramshad, Adekunle M. Adesina, Robert C. Dauser, Jeffrey S. Weinberg, and \\ Murali M. Chintagumpala
}

The University of Texas MD Anderson Cancer Center, Houston, Texas, USA (E.B.L., A.M., A.C.P., L.M.K., D.R.G., M.F.M., S.L.M., J.S.W.); Mayo Clinic, Rochester, Minnesota, USA (A.M.); Texas Children's Cancer Center, Baylor College of Medicine, Houston, Texas, USA (A.C.P., J.Y.J., J.M.S., A.M.A., R.C.D., M.M.C.); Nationwide Children's Hospital, Columbus, Ohio, USA (J.Y.J.); Aarhus University Hospital, Aarhus, Denmark (Y.A.L-R.)

Corresponding Author: Arnold C. Paulino, M.D., Department of Radiation Oncology, MD Anderson Cancer Center, 1515 Holcombe Blvd, Box 97, Houston, TX 77030. (apaulino@mdanderson.org).

\begin{abstract}
Background. Pseudoprogression (PsP) is a recognized phenomenon after radiotherapy (RT) for high-grade glioma but is poorly characterized for low-grade glioma (LGG). We sought to characterize PsP for pediatric LGG patients treated with RT, with particular focus on the role of RT modality using photon-based intensity-modulated RT (IMRT) or proton beam therapy (PBT).

Methods. Serial MRI scans from 83 pediatric LGG patients managed at 2 institutions between 1998 and 2017 were evaluated. PsP was scored when a progressive lesion subsequently decreased or stabilized for at least a year without therapy. Results. Thirty-two patients (39\%) were treated with IMRT, and $51(61 \%)$ were treated with PBT. Median RT dose for the cohort was $50.4 \mathrm{~Gy}(\mathrm{RBE})$ (range, 45-59.4 Gy[RBE]). PsP was identified in 31 patients (37\%), including 8/32 IMRT patients $(25 \%)$ and $23 / 51$ PBT patients $(45 \%)$. PBT patients were significantly more likely to have post-RT enlargement (hazard ratio $[\mathrm{HR}] 2.15,95 \% \mathrm{Cl}: 1.06-4.38, P=0.048$ ). $\mathrm{RT}$ dose $>50.4 \mathrm{~Gy}(\mathrm{RBE})$ similarly predicted higher rates of PsP (HR 2.61, 95\% Cl: $1.20-5.68, P=0.016)$. Multivariable analysis confirmed the independent effects of RT modality $(P=0.03)$ and RT dose $(P=0.01)$ on PsP incidence. Local progression occurred in 10 patients: 7 IMRT patients $(22 \%)$ and 3 PBT patients (6\%), with a trend toward improved local control for PBT patients (HR 0.34, 95\% Cl: $0.10-1.18, P=0.099$ ).

Conclusions. These data highlight substantial rates of PsP among pediatric LGG patients, particularly those treated with PBT. PsP should be considered when assessing response to RT in LGG patients within the first year after RT.
\end{abstract}

\section{Key Points}

1. Pseudoprogression is common following radiotherapy (RT) among pediatric patients with low-grade glioma.

2. Pseudoprogression rates were higher among those treated with proton RT (compared with photon RT), and among those treated with higher doses of RT.

3. Local control is excellent among pediatric low-grade glioma patients treated with RT, irrespective of RT modality.

Pediatric low-grade glioma (LGG), comprising World Health Organization (WHO) grades I and II primary glial neoplasms, can be treated using a variety of therapeutic strategies, including surgery, systemic therapy, and radiotherapy (RT). ${ }^{1,2}$ Due to concerns regarding long-term sequelae, $\mathrm{RT}$ is generally included in the treatment of pediatric LGG 


\section{Importance of the Study}

Pseudoprogression is a recognized phenomenon after RT for high-grade glioma but is poorly characterized for LGG, particularly among pediatric LGG patients. Based on a cohort of 83 pediatric LGG patients, we assessed PsP incidence and kinetics for patients treated with photon-based IMRT and PBT. We found high rates of PsP among the full cohort (37\%), but higher rates of PsP among patients treated with $\mathrm{PBT}$ compared with IMRT (45\% vs $25 \%$ ). Higher RT doses (>50.4 Gy[RBE]) were similarly associated with PsP incidence. Additionally, we observed a trend toward improved local control with PBT versus IMRT. These data highlight substantial rates of PsP among pediatric LGG patients treated with RT, with increased PsP incidence based on RT modality and dose. Given increased utilization of PBT for intracranial pediatric tumors, PsP should be considered when assessing RT response among LGG patients treated with PBT. when the primary tumor is unresectable, recurrent/ progressive, symptomatic, and/or chemo-refractory. ${ }^{1-5}$ When RT is utilized, the 10-year overall survival (OS) for pediatric LGG patients treated is excellent $(>90 \%)$, with 10 -year event-free survival (EFS) rates of approximately $70-75 \% .^{3-5}$

Following RT in the central nervous system, however, prior reports have described a pattern of early post-RT radiographic changes on magnetic resonance imaging (MRI) known as pseudoprogression (PsP). ${ }^{6}$ These radiographic changes can mimic tumor progression, with increased mass effect as well as contrast enhancement for high-grade gliomas. ${ }^{7}$ The phenomenon of PsP is defined by spontaneous improvement in these radiographic changes without oncologic therapy. ${ }^{6-11}$ While PsP among high-grade glioma patients has been relatively well characterized, and appears to be associated with improved disease-related outcomes, the literature regarding PsP incidence among LGG patients, particularly pediatric LGG patients, is sparse. ${ }^{7-12}$ Reports characterizing PsP among LGG patients have reported PsP rates ranging generally between $15 \%$ and $30 \%$ of patients treated with RT. ${ }^{8-13}$

In treating pediatric CNS tumors, RT approaches have evolved. Advanced photon-based RT techniques, such as intensity-modulated RT (IMRT) have been adopted over the last few decades to provide conformal RT treatment plans. ${ }^{5,14}$ However, increased interest has been given to the use of proton beam therapy (PBT) in the treatment of pediatric CNS tumors. ${ }^{14-16}$ The primary advantage of PBT over photon-based approaches such as IMRT centers on the absence of exit dose from a particle beam; this absence of exit dose allows for highly conformal treatment planning with PBT with improved sparing of critical structures. Such considerations are especially important in the treatment of pediatric patients, where minimizing late toxicity of RT is imperative. Consequently, dosimetric and clinical data support the use of PBT in the treatment of pediatric CNS tumors. ${ }^{17-21}$

As pediatric LGG patients are increasingly treated with PBT, the role of RT modality in PsP incidence remains unknown. ${ }^{17-21}$ Data from other CNS lesions, such as ependymoma and atypical teratoid/rhabdoid tumor (AT/RT), suggest substantial rates of radiographic changes on MRI following $\mathrm{PBT}^{22,23}$ We therefore sought to characterize PsP incidence and kinetics, as well as disease-related outcomes, for pediatric LGG patients treated with RT, with a focus on the role of RT modality.

\section{Materials and Methods}

Institutional review board approval was obtained to identify pediatric patients with LGG at 2 institutions who were treated with RT between July 1998 and December 2017. Those with pre-RT MRI studies were included in this study, resulting in identification of 94 patients. Patients with fewer than $3 \mathrm{MRI}$ studies within the first 12 months following RT completion were excluded; this resulted in the exclusion of 11 patients. The medical records for the remaining 83 patients who met inclusion criteria were reviewed for clinical and treatment characteristics, as well as diseaserelated outcomes and treatment-related toxicities. All patients were 18 years of age or younger at time of RT, and all patients had histologically proven LGG.

Two neuroradiologists (J.Y.J. and L.M.K.), blinded to RT modality and RT dose, independently evaluated pre-RT and post-RT follow-up MRI studies, including the T1- and T2-weighted images and T1 images before and after gadolinium contrast administration. Maximal bidimensional measurements of the patients' LGG tumors were provided based on dedicated neuroradiology review of these MRI studies. PsP was scored when the bidirectional product (in $\mathrm{mm}^{2}$ ) of the solid component of tumor increased by at least $5 \%$ of the original pre-RT volume, and the lesion either remained stable or subsequently decreased in size for at least 12 months without new oncologic therapy. The use of the bidimensional tumor product measurements to assess treatment endpoints has been previously validated ${ }^{24}$ and remains in pediatric cooperative group trials for LGG. ${ }^{25}$ Both institutions included in this study utilized for the duration of this study period (through the time of this paper) the bidimensional tumor product to assess tumor status as well as guide clinical decision making. The cutoff of $5 \%$ was selected owing to the largely central location of these tumors (Table 1), and concerns regarding enlarging lesions affecting critical structures such as the optic apparatus and brainstem. Notably, cystic enlargement alone did not result in scoring of PsP in this series. Progression was defined by persistent or progressive tumor growth after RT, resulting in oncologic therapy, clinical symptoms, and/or death. With regard to symptomatic patients, patients with clinical symptoms with persistent or progressive tumor growth following RT were scored as having local progression, whereas patients with clinical symptoms and an enlarging lesion with subsequent spontaneous shrinkage were scored as having symptomatic PsP. 
Table 1 Patient, tumor, and treatment characteristics

\begin{tabular}{|c|c|c|c|c|c|}
\hline & & Full Cohort $(N=83)$ & IMRT $(N=32)$ & PBT $(N=51)$ & $P$-value \\
\hline \multirow[t]{3}{*}{ Age at RT } & Median, y & 10.0 & 8.7 & 10.5 & $P=0.11$ \\
\hline & Range, y & $1.0-17.6$ & $1.0-17.0$ & $3.0-17.6$ & \\
\hline & & $N(\%)$ & $N(\%)$ & $N(\%)$ & \\
\hline \multirow[t]{2}{*}{ Sex } & Male & $46(55)$ & $20(63)$ & $26(51)$ & $P=0.30$ \\
\hline & Female & $37(45)$ & $12(37)$ & $25(49)$ & \\
\hline \multirow[t]{2}{*}{ Histology } & PA & $50(60)$ & $24(75)$ & $26(51)$ & $P=0.03$ \\
\hline & Other LGG & $33(40)$ & $8(25)$ & $25(49)$ & \\
\hline \multirow[t]{2}{*}{ WHO histologic grade } & Grade I & $62(75)$ & $28(88)$ & $34(67)$ & $P=0.03$ \\
\hline & Grade II & $21(25)$ & $4(13)$ & $17(33)$ & \\
\hline \multirow[t]{5}{*}{ Tumor location } & Brainstem & $19(23)$ & $7(22)$ & $12(24)$ & $P=0.36$ \\
\hline & Cerebral hemispheres & $6(7)$ & $0(0)$ & $6(12)$ & \\
\hline & Thalamus & $13(16)$ & $6(19)$ & $7(14)$ & \\
\hline & Optic pathway/hypothalamus & $29(35)$ & $12(38)$ & $17(33)$ & \\
\hline & Other & $16(19)$ & $7(22)$ & $9(18)$ & \\
\hline \multirow[t]{2}{*}{ Tumor lateralization } & Midline & $54(65)$ & $24(75)$ & $30(59)$ & $P=0.26$ \\
\hline & Lateralized & $29(35)$ & $8(25)$ & $21(41)$ & \\
\hline \multirow[t]{3}{*}{ Pretreatment CTV } & Median (cc) & 49.6 & 65.9 & 44.9 & $P=0.51$ \\
\hline & Range (cc) & $4.5-429.2$ & $8.1-282.5$ & $4.5-429.2$ & \\
\hline & & $N(\%)$ & $N(\%)$ & $N(\%)$ & \\
\hline \multirow[t]{3}{*}{ Surgery prior to RT } & GTR & $4(5)$ & $0(0)$ & $4(8)$ & $P=0.18$ \\
\hline & STR & $37(45)$ & $19(59)$ & $18(35)$ & \\
\hline & $\mathrm{Bx}$ & $42(51)$ & $13(41)$ & $29(57)$ & \\
\hline \multirow[t]{2}{*}{ ChT prior to RT } & No & $51(61)$ & $23(72)$ & $28(55)$ & $P=0.12$ \\
\hline & Yes & $32(39)$ & $9(28)$ & $23(45)$ & \\
\hline \multirow{4}{*}{$\begin{array}{l}\text { ChT regimen(s) prior } \\
\text { to } \mathrm{RT}\end{array}$} & Carboplatin/vincristine & $28(34)$ & $9(28)$ & $19(37)$ & \\
\hline & Temozolomide & $5(6)$ & $0(0)$ & $5(10)$ & \\
\hline & Vinblastine & $4(5)$ & $0(0)$ & $4(8)$ & \\
\hline & Other & $9(11)$ & $2(6)$ & $7(14)$ & \\
\hline \multirow[t]{2}{*}{ RT modality } & IMRT & $32(39)$ & $32(100)$ & $0(0)$ & \\
\hline & PBT & $51(61)$ & $0(0)$ & $51(100)$ & \\
\hline \multirow[t]{3}{*}{ RT dose } & Median (Gy[RBE]) & 50.4 & 50.4 & 50.4 & \\
\hline & Range (Gy[RBE]) & $45-59.4$ & $45-59.4$ & $45-54$ & \\
\hline & & $N(\%)$ & $N(\%)$ & $N(\%)$ & \\
\hline \multirow[t]{2}{*}{ RT dose } & $\leq 50.4 \mathrm{~Gy}(\mathrm{RBE})$ & $70(84)$ & $24(75)$ & $46(90)$ & $P=0.06$ \\
\hline & $>50.4 \mathrm{~Gy}(\mathrm{RBE})$ & $13(16)$ & $8(25)$ & $5(10)$ & \\
\hline
\end{tabular}

Includes clinical characteristics by RT modality; chi-squared tests utilized for comparison of proportions by RT modality (IMRT and PBT; $P$-values provided).

Abbreviations: cc, cubic centimeter; Bx, biopsy; ChT, chemotherapy.

RT was delivered using either photon-based IMRT or PBT. CT simulation and custom immobilization were utilized for all patients during RT planning. Anesthesia was utilized for patients too young or unable to tolerate simulation/RT treatments while awake. Of those patients who received PBT $(N=51), 49(96 \%)$ were treated with passive scattering PBT, while $2(4 \%)$ were treated with scanning-beam IM proton therapy. Following RT, included patients were scanned with serial MRI brain studies at least 3 times in the first 12 months postRT. While there was no standardized follow-up regimen for all patients, the typical follow-up included MRI at 2-3 months post-RT, another MRI 4-6 months post-RT, and another MRI 9-12 months post-RT. This regimen was most typical for patients without evidence of increase in tumor size immediately after RT (ie, patients with PsP). For those with increased tumor size in the scans immediately following RT, serial MRI studies were more frequent, generally every 2-3 months until tumor stabilization and/or decrease in size.

Disease-related outcomes including OS, local control (LC), event-free survival (EFS), and PsP incidence were 
calculated. All disease-related outcomes (including OS, LC, EFS, and PsP incidence) were calculated from the end date of RT unless specified otherwise. LC events were defined by disease progression as above; EFS events were defined by disease progression as above or death. PsP incidence events were defined by PsP as above. Disease-related outcomes such as OS, LC, and EFS were calculated using the Kaplan-Meier method. Cumulative incidence curves were generated for PsP incidence. Log-rank test (Cox proportional hazards model) was used to compare survival differences between subgroups of patients defined by disease or treatment characteristics on univariate analysis. Multivariable Cox models using stepwise logistic regression were utilized to provide analysis of multiple predictors of outcome; only significant factors identified on univariate analysis were included in subsequent multivariable models. Testing of the proportional hazards assumption was performed as well to validate described models. Testing of interaction between terms was performed using analysis of variance (ANOVA). Comparison of ratios across groups was performed using chi-squared tests. Comparing PsP kinetic characteristics (such as time-to-peak and timeto-stabilization) were performed between subgroups (ie, RT modality) using $t$-tests. Where relevant, all tests performed were two-sided; $P$-values of $<0.05$ were considered statistically significant. The analyses were performed utilizing SPSS v22.0 and SAS JMP Pro 14.0.0 statistical packages. ${ }^{26,27}$

\section{Results}

\section{Cohort and Treatment Characteristics}

Patient, disease, and treatment characteristics of the 83 pediatric LGG patients treated with RT are summarized in Table 1. Median age at diagnosis was 8.0 years (range, $0.5-$ $17.1 \mathrm{y}$ ), with slightly older age at RT (median, $10.0 \mathrm{y}$; range, 1.0-17.6 years). A majority of patients had midline tumors (53/83, 64\%), with most patients having tumors localized to critical structures such as brainstem and optic pathway/hypothalamus (48/83, 58\%; Table 1). Indications for RT among these 83 patients included progressive disease following prior therapies $(59 / 83,71 \%)$ or high-risk residual disease after either subtotal resection (STR) or biopsy (24/83, 29\%). Less than half of the patients in this cohort were treated with either STR or gross total resection (GTR) before RT, and less than half were treated with pre-RT chemotherapy (Table 1). Among the 32 patients treated with pre-RT chemotherapy, 11 (34\%) received multiple lines of systemic therapy before RT.

With regard to RT modality, 51 patients $(61 \%)$ were treated with PBT, whereas $32(39 \%)$ were treated with IMRT (Table 1). Comparing these 2 cohorts (PBT and IMRT), the proportion of patients with pilocytic astrocytoma (PA) histology tumors was higher among IMRT patients than PBT patients $(75 \%$ vs $51 \%$, chi-squared test, $P=0.03$; Table 1). Similarly, the proportion of patients with WHO grade I tumors (compared with grade II tumors) was higher among IMRT patients ( $88 \%$ vs $67 \%, P=0.03$; Table 1). A trend toward higher RT doses among IMRT patients was further noted, with $25 \%$ IMRT patients receiving $>50.4$ Gy relative biological effectiveness (RBE) compared with $10 \%$ PBT patients $(P=0.06$; Table 1$)$. Otherwise, there were no significant differences between the IMRT and PBT subgroups across a number of other demographic, tumorrelated, and treatment-related characteristics, including with regard to pre-RT extent of resection, pre-RT chemotherapy usage, and clinical tumor volume (CTV) RT target size (Table 1). Also noted was a shorter duration of followup for the PBT patients; as PBT became available at one of the study institutions starting in 2006, PBT patients had a shorter median follow-up than IMRT patients (4.2 vs $6.1 \mathrm{y}, P=0.01)$. Median follow-up for the full cohort was 5.6 years.

\section{Incidence and Predictors of PsP}

PsP was identified in 31 patients (37\%) in the cohort. For patients with PsP, median peak increase in the tumor bidirectional product was 22\% (range, 5-259\%). Among patients with $\mathrm{PsP}$, median time to peak increase in tumor size was 4 months (range, 1-8 mo) following RT; median time from RT to PsP stabilization was 12 months (range 2-39 mo). No influence on PsP incidence was identified based on age at diagnosis, age at RT, sex, grade, tumor location, tumor lateralization, extent of pre-RT surgical resection, pre-RT chemotherapy utilization, and CTV size (all $P>0.05 ;$ Table 2). Histology was found to significantly predict for PsP based on univariate analysis, with a lower incidence of PsP for PA histology versus non-PA LGG histologies (HR 0.47, 95\% confidence interval [Cl] 0.23-0.95, $P=0.037)$.

RT modality was found to predict PsP, with a higher cumulative incidence of PsP among PBT patients (23/51, $45 \%)$ than IMRT patients (8/32, 25\%; Fig. 1). Univariate analysis confirmed the association of RT modality with PsP, with a higher risk of PsP for PBT patients (HR 2.15, 95\% Cl: 1.06-4.38, $P=0.048$; Fig. 1). A test of interaction between RT modality and tumor histology in predicting $\mathrm{Ps} P$ revealed a significant interaction between these variables $(P=0.03)$. Comparing the kinetics of PsP by modality, median time to peak increase in tumor size was similar between IMRT patients (5 mo; range, 1-8 mo) and PBT patients (4 mo; range, $1.5-7 \mathrm{mo} P=0.49$ ) with PsP. The median peak increase in tumor size with $\mathrm{PsP}$ was also comparable between IMRT (17\%; range, 6-259\%) and PBT patients (22\%; range, $5-217 \%$; $P=0.64)$. However, median time from RT to PsP stabilization was longer for IMRT patients (28 mo; range, 6-39 mo) than for PBT patients (12 mo; range, 2-17 mo; $P<0.001)$.

PsP was also predicted by RT dose, with higher RT doses increasing the risk of PsP (Table 2). For patients receiving $>50.4 \mathrm{~Gy}$ (RBE), PsP incidence was $69 \%$ (9/13), compared with $31 \%(22 / 70)$ PsP incidence for those receiving $\leq 50.4$ Gy(RBE) (HR 2.61, 95\% Cl: 1.20-5.68, $P=0.016$; Table 2).

Multivariable analysis (MVA) was then performed to assess PsP risk factors. A model was generated using factors identified on univariate analysis as being significant predictors of PsP, including histology, RT modality, and RT dose (Table 3). Testing the proportional hazards assumption for this MVA model confirmed that the assumption 
Table 2 Univariate predictors of PsP

\begin{tabular}{|c|c|c|c|c|}
\hline & & $\operatorname{PsP}(N=31)$ & No PsP $(N=52)$ & $P$-value \\
\hline \multirow[t]{3}{*}{ Age at RT } & Median, y & 10.9 & 9.5 & $P=0.35$ \\
\hline & Range, y & $3.9-17.0$ & $1.0-17.6$ & \\
\hline & & $N(\%)$ & $N(\%)$ & \\
\hline \multirow[t]{2}{*}{ Sex } & Male & $19(61)$ & $27(52)$ & $P=0.63$ \\
\hline & Female & $12(39)$ & $25(48)$ & \\
\hline \multirow[t]{2}{*}{ Histology } & PA & $14(45)$ & $36(69)$ & $P=0.037$ \\
\hline & Other LGG & $17(55)$ & $16(31)$ & \\
\hline \multirow{2}{*}{$\begin{array}{l}\text { WHO histologic } \\
\text { grade }\end{array}$} & Grade I & $21(68)$ & $41(79)$ & $P=0.23$ \\
\hline & Grade II & $10(32)$ & $11(21)$ & \\
\hline \multirow[t]{5}{*}{ Tumor location } & Brainstem & $7(23)$ & $12(23)$ & $P=0.55$ \\
\hline & Cerebral hemispheres & $2(6)$ & $4(8)$ & \\
\hline & Thalamus & $5(16)$ & $8(15)$ & \\
\hline & Optic pathway/hypothalamus & $8(26)$ & $21(40)$ & \\
\hline & Other & $9(29)$ & $7(13)$ & \\
\hline \multirow[t]{2}{*}{ Tumor lateralization } & Midline & $22(71)$ & $32(62)$ & $P=0.63$ \\
\hline & Lateralized & $9(29)$ & $20(38)$ & \\
\hline \multirow[t]{3}{*}{ Surgery prior to RT } & GTR & $2(6)$ & $2(4)$ & $P=0.68$ \\
\hline & STR & $12(39)$ & $25(48)$ & \\
\hline & $\mathrm{Bx}$ & $17(55)$ & $25(48)$ & \\
\hline \multirow[t]{2}{*}{$\mathrm{ChT}$ prior to $\mathrm{RT}$} & No & $23(74)$ & $28(54)$ & $P=0.11$ \\
\hline & Yes & $8(26)$ & $24(46)$ & \\
\hline \multirow[t]{3}{*}{ RT dose } & Median (Gy[RBE]) & 50.4 & 50.4 & \\
\hline & Range (Gy[RBE]) & $48.6-54$ & $45-59.4$ & \\
\hline & & $N(\%)$ & $N(\%)$ & \\
\hline \multirow[t]{2}{*}{ RT dose } & $\leq 50.4 \mathrm{~Gy}(\mathrm{RBE})$ & $22(71)$ & $48(92)$ & $P=0.016$ \\
\hline & $>50.4 \mathrm{~Gy}(\mathrm{RBE})$ & $9(29)$ & $4(8)$ & \\
\hline \multirow[t]{2}{*}{ RT modality } & IMRT & $8(26)$ & $24(46)$ & $P=0.048$ \\
\hline & PBT & $23(74)$ & $28(54)$ & \\
\hline \multirow[t]{2}{*}{ CTV } & Median (cc) & 49.6 & 53.4 & $P=0.24$ \\
\hline & Range (cc) & $7.6-200.8$ & $4.5-429.2$ & \\
\hline
\end{tabular}

Univariate analyses of predictors of $\mathrm{PsP} ; P$-values provided for univariate tests.

Abbreviations: Bx, biopsy; ChT, chemotherapy; cc, cubic centimeter.

had not been violated $(P=0.44)$. On MVA, PsP remained significantly predicted by RT dose and RT modality (Table 3). However, the effect of histology was not observed on MVA (Table 3).

Most patients with PsP were clinically asymptomatic, and managed with observation and serial MRI studies to follow the course. However, clinical evidence of symptomatic PsP was identified for 6 patients with PsP (6/31, $19 \%$ rate of symptomatic PsP out of all patients with PsP). Five patients with symptomatic PsP were treated with PBT $(5 / 23,22 \%)$, and 1 was treated with IMRT (1/8, 13\%; $P>0.5)$. Rates of symptomatic PsP among the full cohort for each modality were $10 \%$ for PBT $(5 / 51)$ and $3 \%$ for IMRT (1/32). Treatment of symptomatic PsP included steroids for 2 patients, bevacizumab for 1 patient, and surgical intervention for 3 patients. All 3 patients requiring surgical intervention had been treated with PBT; 2 of these patients were treated with repeat resection of the tumor. For both patients undergoing tumor resection for PsP, the indication for surgery was poorly controlled seizures related to the primary lesion; the histologies for these 2 patients were PA and pilomyxoid astrocytoma. Pathology from both surgical specimens after post-RT resection revealed residual glioma with treatment effect; neither patient experienced subsequent regrowth of lesion following post-RT resection. The most severe case of symptomatic PsP was observed in the third patient requiring surgical intervention. This female patient presented at 3.9 years old with a cervicomedullary WHO grade II fibrillary astrocytoma status post initial biopsy of tumor followed by definitive PBT to 50.4 $\mathrm{Gy}(\mathrm{RBE})$; routine MRI 1 month following PBT completion was unremarkable for any radiographic changes. However, 


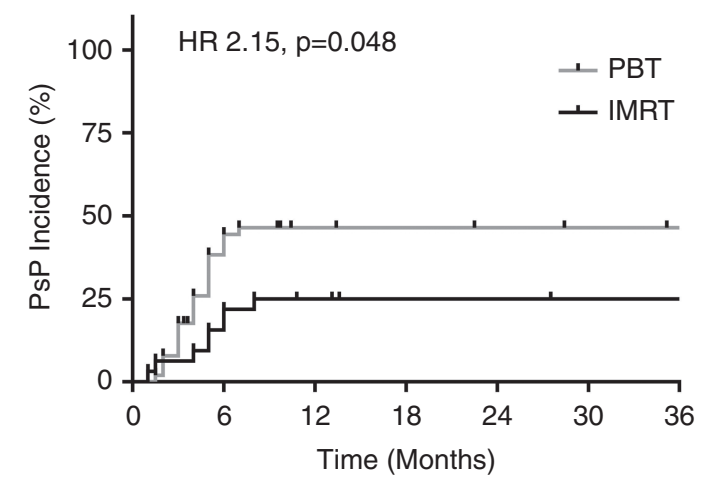

Fig. 1 RT modality predicts for PsP. Cumulative PsP incidence over time by RT modality; significantly increased PsP risk with PBT (gray) versus IMRT (black; HR 2.15, 95\% Cl: 1.06-4.38, $P=0.048$ ).

Table 3 Multivariable model of PsP predictors

\begin{tabular}{llll} 
Factors Included in Model & HR & $95 \% \mathrm{Cl}$ & $P$-value \\
\hline $\begin{array}{l}\text { RT dose (>50.4 Gy[RBE] vs } \leq 50.4 \\
\text { Gy[RBE]) }\end{array}$ & 3.57 & $1.46-8.77$ & $\boldsymbol{P}=\mathbf{0 . 0 0 5}$ \\
\hline RT modality (PBT vs IMRT) & 2.83 & $1.14-7.04$ & $\boldsymbol{P}=\mathbf{0 . 0 2 5}$ \\
\hline Histology (PA vs non-PA LGG) & 0.71 & $0.33-1.53$ & $P=0.378$
\end{tabular}

Model built on the identified significant predictors of PsP on univariate analysis.

2 months following PBT completion, she presented with symptomatic increased intracranial pressure, as well as respiratory failure (Fig. 2). A right frontal shunt was placed to decompress the ventricular system, and patient required respiratory support resulting in prolonged admission and eventually permanent tracheostomy placement. Radiographic PsP peaked at 4 months following PBT, and subsequently stabilized by 12 months post-PBT (Fig. 2). The patient remains alive, without evidence of disease, and with permanent tracheostomy 73 months following completion of PBT.

\section{Outcomes and Predictors of Local Control}

With median follow-up of 5.6 years for the full cohort, 5 -year OS following RT was estimated at 93\% $195 \% \mathrm{Cl}$ : 82-97\%), and 5-year LC at $85 \%(95 \% \mathrm{Cl}: 72-92 \%$; Fig. $3 \mathrm{~A})$. Ten-year OS was found to be $90 \%$ (95\% Cl: $75-96 \%)$, and 10-year LC 82\% (95\% Cl: 69-90\%; Fig. 3A). Local progression was identified in 10 patients $(12 \%)$, at a median time of 39.8 months (range, 4.0-61.1 mo) after completion of RT. Two patients experienced local progression before 12 months after RT completion. Examining LC by RT modality, 7 IMRT patients (22\%) experienced local failure compared with 3 PBT patients (6\%). Five-year LC rates for the IMRT and PBT cohorts were $78 \%$ and $90 \%$, respectively, with a trend toward improved LC with PBT (HR 0.34, 95\% Cl: 0.10-1.18, $P=0.099$; Fig. 3B). Notably, one patient died without evidence of local progression; this patient, with a hypothalamic PA treated with PBT to $45 \mathrm{~Gy}(\mathrm{RBE})$, experienced a hemorrhagic cerebrovascular accident (CVA) 51 months following RT completion. Imaging at the time of CVA demonstrated a hypothalamic tumor still $30 \%$ of the original size of the treated lesion 51 months prior, stable from prior imaging. EFS, which accounts for both local progression and death, therefore revealed no significant benefit of PBT over IMRT (HR 1.79, 95\% Cl: 0.57-5.64, $P=0.32$ ). With regard to the role of PsP on LC, 2 patients with PsP subsequently developed local failure $(6 \%)$, whereas 8 non-PsP patients had local relapse $(15 \%)$; this was not associated with a statistically significant difference in LC by PsP incidence (HR 0.29, 95\% Cl: 0.04-2.39, $P=0.22)$. There was similarly no detected relationship between RT dose and LC (RT dose > 50.4 Gy[RBE], HR $0.61,95 \% \mathrm{Cl}: 0.08-4.81, P=0.64)$, where 1 patient receiving $\mathrm{RT}>50.4 \mathrm{~Gy}$ (RBE) experienced local progression (1/13, $8 \%)$ compared with 9 patients with local failure following RT $\leq 50.4 \mathrm{~Gy}(\mathrm{RBE})(9 / 70,13 \%)$. Additionally, there was no relationship detected between LC and tumor histology (HR $1.20,95 \% \mathrm{Cl}: 0.31-4.65, P=0.79)$ or between LC and WHO histologic grade (HR 1.08, 95\% Cl: 0.23-5.09, $P=0.92$ ).

\section{Discussion}

This series of pediatric LGG patients highlights substantial rates of PsP observed in the months following RT, up to $37 \%$ in the full cohort of patients. Strikingly, we observed significant and independent effects of both RT modality and RT dose on PsP incidence, with higher rates of PsP among PBT-treated patients as well as patients treated with increased RT doses (>50.4 Gy[RBE]). This is particularly relevant in the context of increased utilization of PBT for treatment of pediatric CNS tumors, including LGG. ${ }^{14,28,29}$ With PBT, we further observed a trend toward improved LC compared with photon-based IMRT, raising the possibility of a potential oncologic benefit based on RT modality.

This series is the first to demonstrate a relationship between PsP incidence and RT modality among pediatric LGG patients, and glioma patients more broadly. Prior studies of PBT among other disease sites, including AT/ RT and ependymoma, have suggested rates of post-PBT radiographic changes (including radionecrosis) between $16 \%$ and $43 \% .{ }^{22,23}$ Single-modality series and case reports of PsP after PBT for pediatric LGG have been published as well. ${ }^{30,31}$ Among adult patients LGG (including WHO grades II and III tumors among adults), a recent series including both a photon-treated cohort and a PBT-treated cohort did not demonstrate a difference in PsP rates by $\mathrm{RT}$ modality (14.3\% photon RT vs $16 \%$ PBT). ${ }^{12}$ Notably, most patients from this adult LGG series had tumors with $1 p / 19 q$ codeletion, which has been demonstrated in another adult LGG PsP series to predict lower rates of PsP despite improved prognosis ( $3 \%$ PsP for tumors with codeletion vs $31 \%$ PsP for non-codeleted tumors). ${ }^{10,12}$ Among pediatric LGG tumors, a recent series treated nearly exclusively with photon RT (>95\% of patients) reported a PsP rate of $29 \% .{ }^{11}$ Our series demonstrating a $45 \%$ PsP rate among PBT-treated patients exceeds these previously reported PsP rates from the literature, while the observed $25 \%$ PsP rate among photon-treated patients is comparable to prior photon RT studies. 

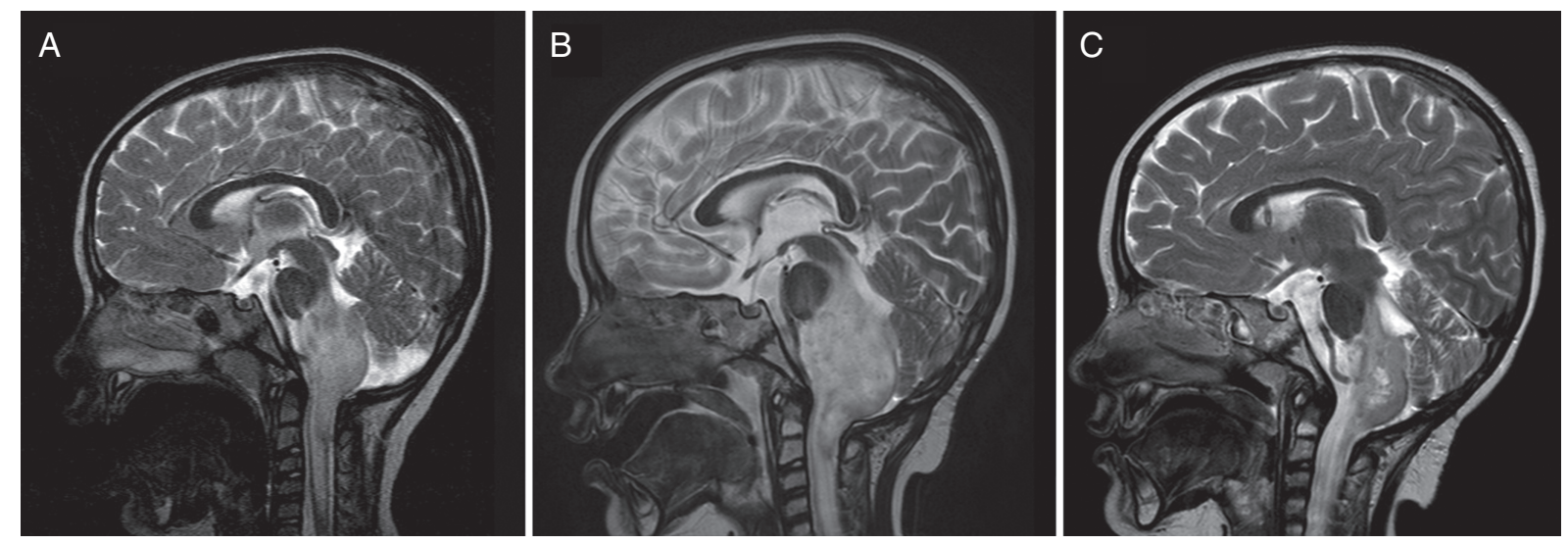

Fig. 2 Case of severe PsP toxicity. A 3.9-year-old female patient presenting with cervicomedullary WHO grade II fibrillary astrocytoma treated with 50.4 Gy(RBE) PBT with subsequent symptomatic PsP requiring ventricular shunt and permanent tracheostomy. T2 mid-sagittal MRI images shown. (A) Pre-PBT baseline scan demonstrates infiltrative cervicomedullary tumor. (B) Four-month post-PBT scan demonstrates increased size of infiltrating tumor (ventricular shunt placed prior to this scan). (C) Twelve-month post-PBT scan demonstrates spontaneous decreased size of tumor; ventricular shunt remains in place.

The mechanism of PsP is thought to be related to underlying RT-induced vascular injuries. ${ }^{32,33}$ Prior studies of pediatric patients treated with PBT for intracranial tumors have demonstrated the association of both subclinical and clinical vasculopathy with increased RT dose. ${ }^{34,35}$ This therefore may account for the observation in this series of higher PsP rates among patients treated with higher RT doses. With regard to RT modality, it is noteworthy that $96 \%$ of PBT-treated patients received passive scattering PBT. Compared with advanced photon-based techniques (such as IMRT), passive scattering PBT has been shown to have increased dose heterogeneity. ${ }^{36}$ With higher risk of dose "hotspots" with passive scattering PBT, regions of high dose may be predisposed to vascular injury, and then subsequent PsP. This hypothesis requires further study, which is under way currently at our institution. Another consideration is that particle beam therapy, including PBT, is generally characterized as having higher radiobiological effectiveness (RBE) than photon RT. ${ }^{37}$ With higher cell-kill effectiveness, clinical concerns have been raised that PBT may increase the risk for radionecrosis, particularly brainstem necrosis, a topic that has received substantial attention within the pediatric oncology community. ${ }^{38-40}$ While no such brainstem radionecrosis events were observed in this series, the clinical literature to date points toward a higher biological effectiveness for PBT. Our group has previously demonstrated that regions of higher linear energy transfer (LET, related to RBE and known to be higher with PBT than photon RT) correspond to specific sites of MRI radiographic changes after $\mathrm{PBT}$ for ependymoma. ${ }^{39}$ It is conceivable that subregions with higher LET correspond to sites of radiographic PsP changes among LGG patients. However, with passive scatter PBT, the high RBE/LET regions tend to fall distal to the target volume (in the direction of the beam ${ }^{41}$; given that these high RBE/LET regions are likely outside the tumor volume, we favor the hypothesis that regions with higher dose, rather than higher RBE/ LET regions, contribute to the vascular injuries that lead to subsequent PsP. Future directions will aim to elucidate those dosimetric parameters associated with PsP.

Looking ahead, assessing those dosimetric parameters associated with PsP during the treatment planning process proves valuable clinically. By knowing regions at higher risk for future PsP, providers may have more confidence that regions of post-RT growth represent PsP rather than local progression. Notably, while most radiographic changes within the first 12 months following RT were a reflection of PsP, 2 patients in this series did experience local progression within the first year after RT. Advanced MRI (as well as advanced positron emission tomography techniques) may provide some insight into differentiating PsP from true progression. ${ }^{42,43}$ However, these methods have yet to be widely validated and adopted. Rather, examining dose inhomogeneity or variable RBE/LET during PBT planning process may allow for the superimposition of these maps onto posttreatment MRI studies. ${ }^{44,45}$ Should regions of higher dose or higher LET correspond to sites with increased risk for PsP, this could facilitate differentiation between PsP and true progression on posttreatment imaging. Furthermore, while rates of symptomatic PsP in the present series were relatively low (19\%, compared with prior series reporting $32 \%$ rate of symptomatic $\mathrm{PsP}),{ }^{11}$ surgical intervention was required in 3 patients, including 1 needing permanent tracheostomy. Particularly in the context of higher PsP risk with PBT, treatment planning taking into account factors associated with PsP could allow for a priori treatment planning modifications to prevent highdose or high-LET regions from being adjacent to or involving critical structures.

Along the same lines, with higher rates of PsP among PBT-treated patients, we observed a trend toward improved LC with PBT. Among high-grade glioma patients (both children and adults), PsP predicts improved diseaserelated outcomes. ${ }^{6,46-48}$ Among LGG patients, this relationship is not as well defined, with reports conflicting regarding the interaction between $\mathrm{PsP}$ and outcome..$^{8,11,12}$ 


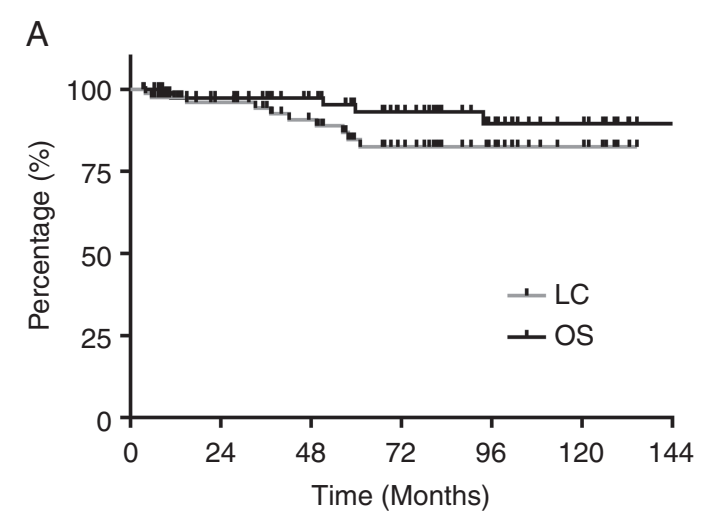

B

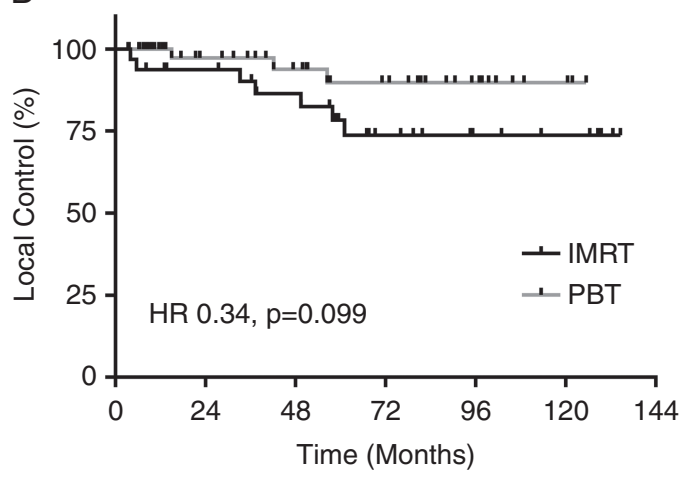

Fig. 3 Disease-related outcomes. (A) Kaplan-Meier survival curves for overall survival (OS, black) and local control (LC, gray) for the full cohort. (B) Kaplan-Meier survival curves for LC by RT modality; trend toward improved LC for patients treated with PBT (gray) versus IMRT (black; HR 0.34, 95\% Cl: 0.10-1.18, $P=0.099$ ).

In this retrospective series, we did not observe a significant relationship between PsP incidence and LC, with 2 patients developing local progression years after initial PsP following RT. However, larger studies may be sufficiently powered to identify a role for PsP in predicting outcomes. Conceptually, there is a hypothetical mechanism by which use of PBT improves disease-related outcomes (including $\mathrm{LC}$ ); with increased vascular injury, $\mathrm{PBT}$ results in higher rates of $\mathrm{PsP}$, which in turn translate into lower incidence of local failure. Institutions should be encouraged to report their experiences in the treatment of pediatric LGG with $P B T$ to better assess this possible relationship between RT modality and LC among larger series. It is also worth noting that the relationship between RT modality and disease-related outcomes was complicated after inclusion of a patient treated with PBT who developed a subsequent CVA without evidence of local failure (that is, analyzing EFS as a function of RT modality rather than LC as a function of RT modality). This CVA may represent a late vascular complication of RT (in this case, PBT), supported by literature reporting rates of serious vasculopathy after PBT for pediatric brain tumor patients as high as $2.6 \%$ at 3 years. ${ }^{34}$ With this in mind, drawing conclusions regarding $\mathrm{PBT}$ conferring improved outcomes should be tempered, and further studies are necessary to assess the overall impact of RT modality on outcomes, and not solely local control.

The role of histology has also been discussed as regards PsP following RT for pediatric LGG. Two series, both utilizing photon-based $R T$, have reported significantly higher rates of PsP among patients with PA histologies (PsP incidence $41-65 \%$ for PA patients). ${ }^{9,11}$ Notably, in both series, cystic enlargement was included in the definition of PsP, whereas only solid component enlargement was included in the definition of PsP in the present study. This definition of PsP, defined a priori for this series, reflected the underlying proposed mechanism of PsP, which centers on increased vascular permeability and injury in solid tumor components. ${ }^{32,33}$ On initial univariate analysis, our data suggested that non-PA histologies had higher incidence of PsP, which was not supported on MVA; this is explained by the larger proportion of non-PA histologies among PBTtreated patients. While cystic enlargement (particularly among PA patients) can occur following RT, our definition of PsP reported here reflects the proposed mechanism of the PsP phenomenon, and our data suggest that a solidcomponent-centric definition of PsP does not result in differential PsP incidence by histology. Separate studies investigating cystic changes (among PA patients as well as patients with cystic nonglial tumors such as craniopharyngiomas) should be pursued, with focus on the role of RT modality on these cystic changes.

This study includes a number of limitations. The definition of PsP utilized in this study relies on the bidimensional tumor product, which has been previously described in the context of pediatric LGG cooperative group studies. ${ }^{24,25}$ The bidimensional tumor product was used at both institutions in this series both to assess tumor response and to guide clinical decision making. However, the cutoff of $5 \%$ increase in bidimensional product, which was decided a priori, has not been previously validated. The basis for this cutoff was the hypothesis that even small increases in the tumor size may cause symptoms given the proximity of tumors to critical structures; in this series, a majority of patients had midline tumors, and similarly over half of the patients in this series had tumors located at the brainstem, optic pathway, or hypothalamus. The rationale for this cutoff was supported in this series, as patients with symptomatic PsP had bidimensional tumor product increases of as little as $8 \%$. That said, further studies to validate this PsP definition are needed. Additionally, 11 patients were excluded from the cohort due to inadequate follow-up imaging, a potential source of bias or missed events. Seven of these patients were treated with PBT, and 4 were treated with IMRT. All were from outside of the Houston, Texas area, the only observed common trend among these patients. We therefore speculate that exclusion of these patients is not a significant source of bias in this series. As a retrospective series, there are further limitations associated with reviewing records of patients treated over a two-decade period. It is possible that IMRT-treated patients, who were treated earlier, may have had poorer-quality MRI studies, leading to inferior target volume delineation during the planning process and associated higher risk of local failure. This could represent a potential confound to account for the trend toward poorer LC among IMRT patients. Examining other 
factors, we found no difference between the IMRT and PBT cohorts as regards grade, treated tumor volume size (CTV), or frequency of posttreatment MRI surveillance $(P=0.51)$. Lastly, molecular testing for these patients, including tumor BRAF or $\mathrm{H} 3$ histone mutational status, was not available for this analysis; future studies will endeavor to include this molecular information to identify potential molecular risk factors for outcomes such as PsP.

Collectively, the disease-related outcomes for pediatric LGG patients treated with RT are excellent in this cohort, with 10-year OS and LC rates comparable to other published data. ${ }^{3-5}$ The data are particularly reassuring for the use of PBT in the treatment of pediatric LGG, where longterm LC rates (90\% 10-year LC for PBT-treated patients) mirror those for photon RT-treated patients in large prior studies. ${ }^{3-5}$ Therefore, PBT should be considered a reasonable option in the context of RT for pediatric $\mathrm{LGG}^{28,29}$; providers recommending PBT should be aware of the increased risk of PsP after RT as above. Looking ahead, these data raise the question of optimal RT modality and dose for treatment of these challenging cases. Further follow-up continuing exploration of the late RT-related sequelae by RT modality and RT dose will be critical to addressing this question. Our joint institutional practice, supported by these data, remains to continue treatment of pediatric LGG patients with PBT to doses $\leq 50.4 \mathrm{~Gy}(\mathrm{RBE})$.

\section{Keywords}

low-grade glioma | pediatric malignancy | proton beam therapy | pseudoprogression | radiotherapy

\section{Funding}

No funding supported this research.

Conflict of interest statement. All authors report no conflicts of interest or relevant financial disclosures related to this work.

Authorship statement. Ethan B. Ludmir: conceptualization, data curation, formal analysis, investigation, visualization, original draft, writing, reviewing, and editing. Anita Mahajan: conceptualization, data curation, formal analysis, investigation, original draft, writing, reviewing, and editing. Arnold C. Paulino: conceptualization, data curation, investigation, original draft, writing, reviewing, and editing. JeremyY. Jones: conceptualization, data curation, investigation, writing, reviewing, and editing. Leena M. Ketonen: conceptualization, data curation, investigation, writing, reviewing, and editing. Jack $M$. Su: conceptualization, investigation, original draft, writing, reviewing, and editing. David R. Grosshans: conceptualization, investigation, writing, reviewing, and editing. Mary Frances McAleer: conceptualization, investigation, writing, reviewing, and editing. Susan L. McGovern: conceptualization, investigation, writing, reviewing, and editing. Yasmin Lassen-Ramshad: conceptualization, investigation, writing, reviewing, and editing. Adekunle M. Adesina: conceptualization, data curation, investigation, writing, reviewing, and editing. Robert C. Dauser: conceptualization, investigation, writing, reviewing, and editing. Jeffrey S. Weinberg: conceptualization, investigation, writing, reviewing, and editing. Murali M. Chintagumpala: conceptualization, data curation, investigation, visualization, original draft, writing, reviewing, and editing.

Presentations. Presented in part at the International Society of Pediatric Neuro-Oncology (ISPNO) Conference in Denver, CO (2018).

\section{References}

1. Oaddoumi I, Sultan I, Gajjar A. Outcome and prognostic features in pediatric gliomas: a review of 6212 cases from the Surveillance, Epidemiology, and End Results database. Cancer. 2009;115(24):5761-5770.

2. Packer RJ, Ater J, Allen J, et al. Carboplatin and vincristine chemotherapy for children with newly diagnosed progressive low-grade gliomas. J Neurosurg. 1997;86(5):747-754.

3. Merchant TE, Kun LE, Wu S, Xiong X, Sanford RA, Boop FA. Phase II trial of conformal radiation therapy for pediatric low-grade glioma. J Clin Oncol. 2009;27(22):3598-3604.

4. Gnekow AK, Falkenstein F, von Hornstein S, et al. Long-term followup of the multicenter, multidisciplinary treatment study HIT-LGG-1996 for low-grade glioma in children and adolescents of the German Speaking Society of Pediatric Oncology and Hematology. Neuro Oncol. 2012;14(10):1265-1284.

5. Paulino AC, Mazloom A, Terashima K, et al. Intensity-modulated radiotherapy (IMRT) in pediatric low-grade glioma. Cancer. 2013;119(14): 2654-2659

6. Gunjur A, Lau E, Taouk Y, Ryan G. Early post-treatment pseudo-progression amongst glioblastoma multiforme patients treated with radiotherapy and temozolomide: a retrospective analysis. J Med Imaging Radiat Oncol. 2011;55(6):603-610.

7. Brandsma D, Stalpers L, Taal W, Sminia P, van den Bent MJ. Clinical features, mechanisms, and management of pseudoprogression in malignant gliomas. Lancet Oncol. 2008;9(5):453-461.

8. van West SE, de Bruin HG, van de Langerijt B, Swakk-Kragten AT, van den Bent MJ, Taal W. Incidence of pseudoprogression in low-grade gliomas treated with radiotherapy. Neuro Oncol. 2017;19(5):719-725.

9. Naftel RP, Pollack IF, Zuccoli G, Deutsch M, Jakacki RI. Pseudoprogression of low-grade gliomas after radiotherapy. Pediatr Blood Cancer. 2015;62(1):35-39.

10. Lin AL, Liu J, Evans J, et al. Codeletions at $1 p$ and 19q predict a lower risk of pseudoprogression in oligodendrogliomas and mixed oligoastrocytomas. Neuro Oncol. 2014;16(1):123-130. 
11. Tsang DS, Murphy ES, Lucas JT Jr, Lagiou P, Acharya S, Merchant TE. Pseudoprogression in pediatric low-grade glioma after irradiation. $J$ Neurooncol. 2017;135(2):371-379.

12. Bronk JK, Guha-Thakurta N, Allen PK, Mahajan A, Grosshans DR, McGovern SL. Analysis of pseudoprogression after proton or photon therapy of 99 patients with low grade and anaplastic glioma. Clin Trans/ Radiat Oncol. 2018:9:30-34.

13. Mannina EM, Bartlett GK, McMullen KP. Extended volumetric follow-up of juvenile pilocytic astrocytomas treated with proton beam therapy. Int J Particle Ther. 2016;3(2):291-299.

14. Khan AJ, Kann BH, Pan W, Drachtman R, Roberts K, Parikh RR. Underutilization of proton therapy in the treatment of pediatric central nervous system tumors: an analysis of the national cancer database. Acta Oncol. 2017;56(8):1122-1125.

15. Ladra MM, MacDonald SM, Terezakis SA. Proton therapy for central nervous system tumors in children. Pediatr Blood Cancer. 2018;65(7):e27046.

16. Chhabra A, Mahajan A. Treatment of common pediatric CNS malignancies with proton therapy. Chin Clin Oncol. 2016;5(4):49.

17. Harrabi SB, Bougatf N, Mohr A, et al. Dosimetric advantages of proton therapy over conventional radiotherapy with photons in young patients and adults with low-grade glioma. Strahlenther Onkol. 2016;192(11):759-769.

18. Dennis ER, Bussiere MR, Niemierko A, et al. A comparison of critical structure dose and toxicity risks in patients with low grade gliomas treated with IMRT versus proton radiation therapy. Technol Cancer Res Treat. 2013;12(1):1-9.

19. Greenberger BA, Pulsifer MB, Ebb DH, et al. Clinical outcomes and late endocrine, neurocognitive, and visual profiles of proton radiation for pediatric low-grade gliomas. Int J Radiat Oncol Biol Phys. 2014;89(5):1060-1068.

20. Kahalley LS, Ris MD, Grosshans DR, et al. Comparing intelligence quotient change after treatment with proton versus photon radiation therapy for pediatric brain tumors. J Clin Oncol. 2016;34(10): 1043-1049.

21. Eaton BR, Esiashvili N, Kim S, et al. Endocrine outcomes with proton and photon radiotherapy for standard risk medulloblastoma. Neuro Oncol. 2016:18(6):881-887.

22. Gunther JR, Sato M, Chintagumpala M, et al. Imaging changes in pediatric intracranial ependymoma patients treated with proton beam radiation therapy compared to intensity modulated radiation therapy. Int $J$ Radiat Oncol Biol Phys. 2015;93(1):54-63.

23. McGovern SL, Okcu MF, Munsell MF, et al. Outcomes and acute toxicities of proton therapy for pediatric atypical teratoid/rhabdoid tumor of the central nervous system. Int J Radiat Oncol Biol Phys. 2014;90(5):1143-1152.

24. Macdonald DR, Cascino TL, Schold SC Jr, Cairncross JG. Response criteria for phase II studies of supratentorial malignant glioma. J Clin Oncol. 1990:8(7):1277-1280.

25. Ater JL, Zhou T, Holmes E, et al. Randomized study of two chemotherapy regimens for treatment of low-grade glioma in young children: a report from the Children's Oncology Group. J Clin Oncol. 2012;30(21):2641-2647.

26. IBM Corp. IBM SPSS Statistics for Windows, Version 22.0. Armonk, NY: IBM Corp.; 2013

27. SAS Institute Inc. SAS JMP Pro for Windows, Version 14. Cary, NC: SAS Corp; 2018.

28. Shih HA, Sherman JC, Nachtigall LB, et al. Proton therapy for low-grade gliomas: Results from a prospective trial. Cancer. 2015:121(10):1712-1719.

29. Hauswald $H$, Rieken $S$, Ecker $S$, et al. First experiences in treatment of low-grade glioma grade I and II with proton therapy. Radiat Oncol. 2012;7:189.
30. Lassen-Ramshad Y, Petersen JB, Tietze A, Borghammer P, Mahajan A, McGovern SL. Pseudoprogression after proton radiotherapy for pediatric low grade glioma. Acta Oncol. 2015;54(9):1701-1702.

31. Meyzer C, Dhermain F, Ducreux D, et al. A case report of pseudoprogression followed by complete remission after proton-beam irradiation for a low-grade glioma in a teenager: the value of dynamic contrast-enhanced MRI. Radiat Oncol. 2010;5:9.

32. Rider WD. Radiation damage to the brain-a new syndrome. J Can Assoc Radiol. 1963;14:67-69.

33. Parvez K, Parvez A, Zadeh G. The diagnosis and treatment of pseudoprogression, radiation necrosis and brain tumor recurrence. Int J Mol Sci. 2014;15(7):11832-11846.

34. Hall MD, Bradley JA, Rotondo $R L$, et al. Risk of radiation vasculopathy and stroke in pediatric patients treated with proton therapy for brain and skull base tumors. Int J Radiat Oncol Biol Phys. 2018;101(4):854-859.

35. Kralik SF, Mereniuk TR, Grignon L, et al. Radiation-Induced Cerebral Microbleeds in Pediatric Patients With Brain Tumors Treated With Proton Radiation Therapy. Int J Radiat Oncol Biol Phys. 2018. pii: S03603016(18):33484-9. doi:10.1016/j.jijrobp.2018.07.2016.

36. Freund D, Zhang R, Sanders M, Newhauser W. Predictive risk of radiation induced cerebral necrosis in pediatric brain cancer patients after VMAT versus proton therapy. Cancers (Basel). 2015;7(2):617-630.

37. Abolfath R, Peeler CR, Newpower M, Bronk L, Grosshans D, Mohan R. A model for relative biological effectiveness of therapeutic proton beams based on a global fit of cell survival data. Sci Rep. 2017;7(1):8340.

38. Haas-Kogan D, Indelicato D, Paganetti $H$, et al. National cancer institute workshop on proton therapy for children: considerations regarding brainstem injury. Int J Radiat Oncol Biol Phys. 2018;101(1):152-168.

39. Peeler CR, Mirkovic D, Titt U, et al. Clinical evidence of variable proton biological effectiveness in pediatric patients treated for ependymoma. Radiother Oncol. 2016;121(3):395-401.

40. Indelicato DJ, Flampouri S, Rotondo RL, et al. Incidence and dosimetric parameters of pediatric brainstem toxicity following proton therapy. Acta Oncol. 2014;53(10):1298-1304.

41. Sanchez-Parcerisa D, Cortés-Giraldo MA, Dolney D, Kondrla M, Fager M, Carabe A. Analytical calculation of proton linear energy transfer in voxelized geometries including secondary protons. Phys Med Biol. 2016:61(4):1705-1721.

42. Ma B, Blakeley JO, Hong $X$, et al. Applying amide proton transferweighted MRI to distinguish pseudoprogression from true progression in malignant gliomas. J Magn Reson Imaging. 2016;44(2):456-462.

43. Galldicks N, Dunkel V, Stoffels G, et al. Diagnosis of pseudoprogression in patients with glioblastoma using 0-(2-[-18 F]fluoroethyl)-L-tyrosine PET. Eur J Nucl Med Mol Imaging. 2015;42:685-95.

44. Guan F, Peeler C, Bronk L, et al. Analysis of the track- and dose-averaged LET and LET spectra in proton therapy using the geant4 Monte Carlo code. Med Phys. 2015;42(11):6234-6247.

45. Cao W, Khabazian A, Yepes PP, et al. Linear energy transfer incorporated intensity modulated proton therapy optimization. Phys Med Biol. 2017;63(1):015013.

46. Carceller F, Fowkes LA, Khabra K, et al. Pseudoprogression in children, adolescents and young adults with non-brainstem high grade glioma and diffuse intrinsic pontine glioma. J Neurooncol. 2016;129(1):109-121.

47. Kang $\mathrm{HC}, \mathrm{Kim} \mathrm{CY}, \mathrm{Han} \mathrm{JH}$, et al. Pseudoprogression in patients with malignant gliomas treated with concurrent temozolomide and radiotherapy: potential role of p53. J Neurooncol. 2011;102(1):157-162.

48. Gahramanov S, Varallyay C, Tyson RM, et al. Diagnosis of pseudoprogression using MRI perfusion in patients with glioblastoma multiforme may predict improved survival. CNS Oncol. 2014;3(6):389-400 